INTERNATIONAL JOURNAL OF AGRICULTURE \& BIOLOGY

ISSN Print: $1560-8530$; ISSN Online: $1814-9596$

17-0264/2017/19-5-1119-1124

DOI: $10.17957 / \mathrm{IJAB} / 15.0393$

http://www.fspublishers.org

Full Length Article

\title{
Effects of Drought Stress on Growth and Development of Wheat Seedlings
}

\author{
Hongying Duan, Yanqiu Zhu', Jingyun $\mathrm{Li}^{\dagger}$, Weikai Ding, Huina Wang, Lina Jiang and Yanqing Zhou* \\ Henan Normal University, College of Life Sciences, XinXiang, 453007, China \\ *For correspondence: dxdhy@163.com,yqzhou@htu.cn \\ †These authors made equal contributions to this work
}

\begin{abstract}
In this study, two wheat cultivars XM13 and AK58 under different drought tolerance were compared by evaluating seed germination, seedling growth and microstructures in order to identify the drought resistance of two wheat cultivars. Results showed that the germination of wheat seeds could be inhibited by drought stress, the germination energy significantly decreased with the increase of drought degree, and the germination energy of AK58 was significantly stronger compared with XM13. Although the root length of wheat XM13 were little influenced by drought stress, AK58 can be promoted and appeared significant differences with the control except the stress of $25 \% \mathrm{PEG}_{6000}$. Along with the increase of drought degree, the volume of cortex cell in root had a trend of decline, even shrank at the stress of $15-25 \% \mathrm{PEG}_{6000}$, but effect of drought stress on root growth of wheat XM13 was especially significant compared with wheat AK58. In addition, leaf area of wheat seedling decreased under drought stress, but the decreasing amplitude of wheat AK58 leaf area was greater than XM13. Further study identified that palisade tissue cell in leaf gradually became shorter and disorder, and the thickness of spongy tissue decreased, which was especially significant under the stress of 20-25\% PEG 6000 . Compared with wheat XM13, the atrophy of endodermis cell in leaf of wheat AK58 should be later. Therefore, the drought-resistant ability of two cultivars wheat was different, and wheat AK58 might be stronger and could better adapt to drought stress compared with wheat XM13. (C) 2017 Friends Science Publishers
\end{abstract}

Keywords: Triticum aestivum; Drought stress; Leaf; Root; Microstructure

\section{Introduction}

In recent years, drought is one of the most common abiotic factors which harmed growth and development of plant, and is becoming an increasingly severe problem in many regions of the world (Zalibekov, 2011; Hossain et al., 2013; Lipiec et al., 2013). Extreme water shortage causes considerable physiological, metabolic and morphological changes in plant, and ultimately reduces crop yield and quality (Maqsood et al., 2012). However, there are some protection mechanisms in plant which could be beneficial to plant in response to drought stress. Under drought stress, the morphology, photosynthesis, antioxidant enzymes or osmotic adjustment substances in a plant would be adversely influenced and changed. The severity of drought depends on the growth stage of plant (Maqsood et al., 2013). In addition, root is the key organ of plant against drought, because it could absorb water and nutrients from soil (Cseuz et al., 2009; Shen et al., 2013). The response of root growth to water deficits is important in drought tolerance (Carvalho et al., 2014; Wang et al., 2014a). Under drought stress, leaf of plant could also protect water and improve utilization rate of water. Therefore, the morphology of plant could show a variety of strategies to adapt to drought stress, which is important for plant to survive and grow under drought stress (Bhargava and Sawant, 2013; Grzesiak et al., 2013).

Wheat (Triticum aestivum L.) is one of the important crops in the world, contains starch, protein, sugar and provides food for human population (Peleg et al., 2011; Liu et al., 2016; Yu et al., 2016). At present, drought stress has become the main one in abiotic stresses, and could restrain growth and yield of wheat (Lopes and Reynolds, 2010; Boscaiu et al., 2011; Gong, 2014). According to the Food and Agriculture Organization of the United Nations, Human farming, grazing took possession of $25-30 \%$. Humans cannot effectively use the remaining dry land for plant production (Hu and Xiong, 2014; Zhang et al., 2016). Thus, it is urgent to clarify effect of drought stress on growth and development of wheat. Polyethylene glycol (PEG) is nonionic, inert, non-penetrating and osmotically active polymer, could induce dehydration by decreasing water potential of soil, and so is generally used to simulate soil water environment of plants such as wheat (Ramya et al., 2016), rice (Ma et al., 2015) and maize (Khodarahmpour and Motamedi, 2001). In this research, seed germination, 
seedling growth and microstructure of two wheat cultivars under drought stress were studied to explore effect mechanism of drought stress on wheat seedlings, which would provide theoretical basis for breeding and evaluation of drought-resistant wheat.

\section{Materials and Methods}

\section{Plant Materials}

Wheat seeds (Triticum aestivum) cultivar, Xinmai13 (XM13) and Aikang58 (AK58) were respectively provided by Xinxiang Academy of Agricultural Sciences and Henan Institute of Science and Technology, P. R. China.

\section{Germination of Wheat Seeds}

Wheat seeds were disinfected for $10 \mathrm{~min}$ in beaker with $0.1 \% \mathrm{HgCl}_{2}$, running water for $50 \mathrm{~min}$ and then washed 3-5 times with distilled water. 100 wheat seeds of each group were grown in $12 \mathrm{~cm}$ sterile petri dishes containing two

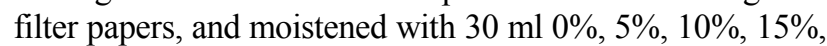
$20 \%$ and 25\% PEG $_{6000}$ (Yan, 2015), respectively. Subsequently, the petri dish was placed in the incubator at $24 \pm 1^{\circ} \mathrm{C}$ with $45 \%$ relative humidity, and $14 / 10 \mathrm{~h}$ light/dark photoperiod (4500 Lux) except for the first three days. The germination energy (GE) and germination rate (GR) of wheat seeds were respectively calculated after sown in hydroponics culture for $4 \mathrm{~d}$ and $8 \mathrm{~d}$ (Jung et al., 2006; El Harfi et al., 2016), GE $=(\mathrm{N} 4 / 100) \times 100, \mathrm{GR}=(\mathrm{N} 8 / 100)$ $\times 100$, $N 4=$ number of seed germinated on the 4 day, $N 8=$ number of seed germinated on the 8 day. In addition, there were three replications for each group.

\section{Cultivation of Wheat Seedlings}

Wheat seeds were sown in $15 \mathrm{~cm}$ pots with the equal mixture of soil, peat and vermiculite, and irrigated with distilled water once every 2 days, then were kept at $24 \pm 1^{\circ} \mathrm{C}$ in the light $(14 / 10 \mathrm{~h}$ light/dark photoperiod, 4500LUX). Seven days later, irrigated with $100 \mathrm{~mL} 0 \%, 5 \%, 10 \%, 15 \%$, $20 \%$ or $25 \% \mathrm{PEG}_{6000}$, respectively. At the three-leaf stage, growth of wheat seedling was observed, root length was measured from the collar to the root tip, and the leaf area was measured by length-width method. In addition, there were 300 wheat seeds in each group with three replications.

\section{Preparation of Paraffin Section}

Small pieces of $0.2 \mathrm{~cm} \times 0.5 \mathrm{~cm}$ were cut off from root and leaf of wheat seedling in three-leaf stage, and were quickly fixed with 50\% FAA solution for more than $48 \mathrm{~h}$. After, these pieces were processed as the followings: dehydration by a series of gradient-concentration ethanol, transparence with gradient-concentration xylene, immersion and embedment with paraffin. Subsequently, the tissue sample was sliced by microtome, the thickness of transection was $10 \mu \mathrm{m}$, and then paraffin section was observed by Olympus photo microscope (Duan et al., 2009; Wang et al., 2014b). In addition, there was repeated three times for each tissue sample in this research.

\section{Statistics Analysis of Data}

The results were analyzed by analysis of variance (ANOVA) and multiple comparisons of least significant difference (LSD) using SPSS software.

\section{Results}

\section{Effect of Drought Stress on Germination of Wheat Seeds}

Compared with the control, the germination energy and germination rate of two wheat cultivars were low, and reduced significantly with the increased concentration of $\mathrm{PEG}_{6000}$ (Fig. 1; Fig. 2). Under the normal condition, the germination energy of wheat XM13 was greater than wheat AK58, however was significantly lower than wheat AK58 under the stress of $\mathrm{PEG}_{6000}$. The germinate rate of seed treated with $25 \% \mathrm{PEG}_{6000}$ was higher in wheat AK58 than wheat XM13, which was $26.7 \%$ and $16.7 \%$, respectively, and significantly decreased compared with the control. The result indicated that seed germination was inhibited by $\mathrm{PEG}_{6000}$, and the drought resistant ability of wheat AK58 was stronger than wheat XM13.

\section{Effect of Drought Stress on Growth of Wheat Seedlings}

Under the normal condition, root length of wheat XM13 was longer than wheat AK58, however shorter than wheat AK58 under drought stress (Fig. 3). Compared to the control, root length of wheat AK58 increased and showed a trend of inverted bell along with the increase of PEG 6000 concentration, but root length of wheat XM13 had no significant change. As stressed with $10 \% \mathrm{PEG}_{6000}$, the root of wheat AK58 was the longest and about $16.21 \mathrm{~cm}$, and appeared extremely significant compared with the control. In addition, under the stress of $25 \% \mathrm{PEG}_{6000}$, root length of wheat AK58 was only $11.51 \mathrm{~cm}$ and also longer compared with the control.

Under the normal condition, leaf area of wheat AK58 was significantly greater than wheat XM13 (Fig. 4). Compared with control, leaf area of wheat AK58 decreased and became smaller with the increasing concentration of $\mathrm{PEG}_{6000}$. As treated with $15 \%, 20 \%$ and $25 \% \mathrm{PEG}_{6000}$, leaf area of wheat AK58 was respectively $3.5 \mathrm{~cm}^{2}, 2.05 \mathrm{~cm}^{2}$ or $1.68 \mathrm{~cm}^{2}$, and reached a significant level compared with the control. Although leaf area of wheat XM13 was about 5.975 $\mathrm{cm}^{2}$ at $5 \% \mathrm{PEG}_{6000}$ and larger than the control, decreased gradually along with the increasing concentration of $\mathrm{PEG}_{6000}$, even was smaller under the stress of $15-25 \%$ $\mathrm{PEG}_{6000}$ and reached significant difference with the control. 


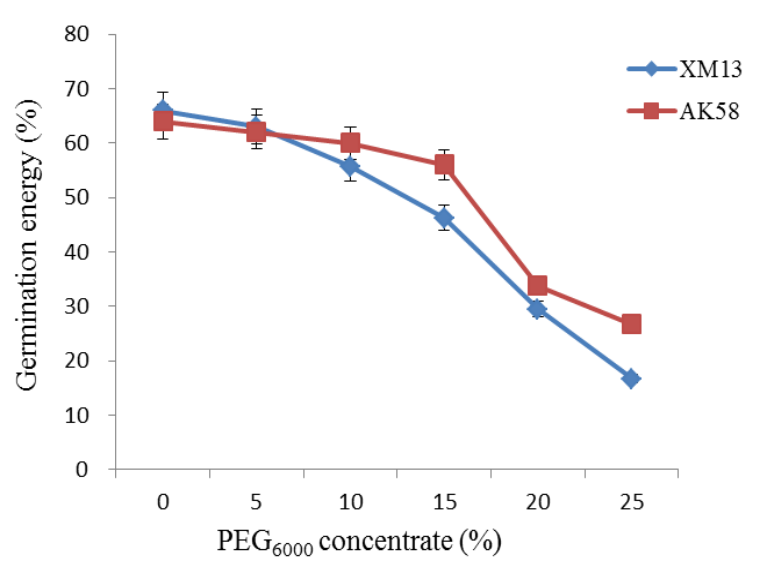

Fig. 1: The germination energy of wheat seeds under drought stress. The error bars represented standard errors

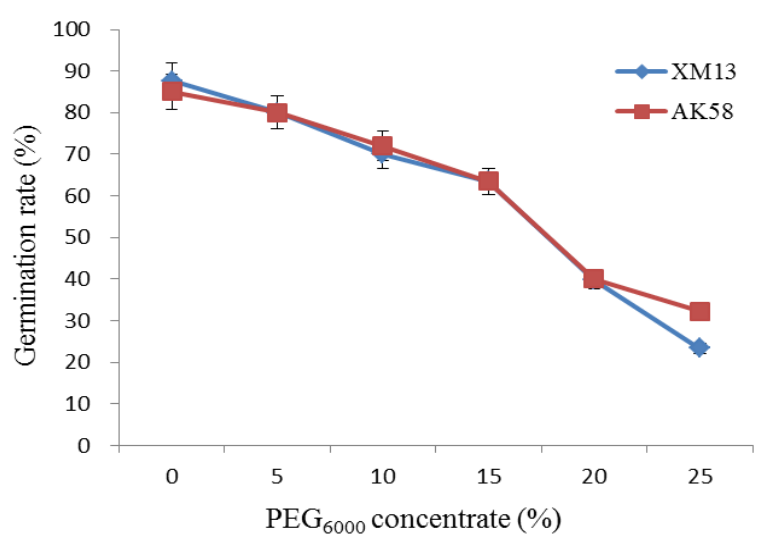

Fig. 2: The germination rate of wheat seeds under drought stress. The error bars represented standard errors

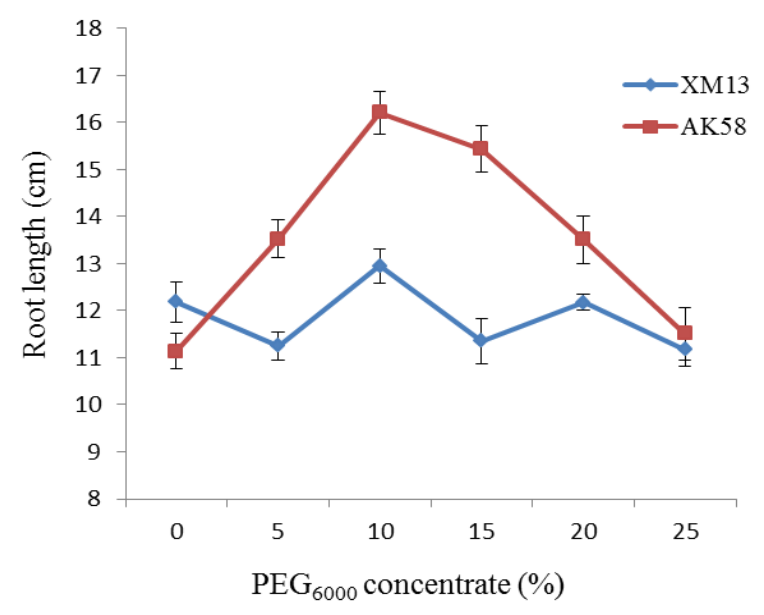

Fig. 3: The root length of wheat seedling under drought stress. The error bars represented standard errors

Thus, the leaf area of wheat seedling reduced under drought stress, and the decreasing amplitude of wheat AK58 was greater than wheat XM13.

\section{Effect of Drought Stress on Microstructure of Wheat Seedlings}

Under the normal condition, the volume of exodermal cell in seedling roots of wheat XM13 and AK58 was large, cortical parenchyma cell and endodermal cell were full, and the main xylem vessels were circular (Fig. 5a; 6a). Furthermore the difference was not significant between two wheat cultivars. Compared with the control, the volume of endodermal cell in root of wheat XM13 reduced as treated with $10 \%$ PEG $_{6000}$ (Fig. 5c), but changes of endodermal cell in root of wheat AK58 were not obvious (Fig. 6c). Under the stress of $15 \% \mathrm{PEG}_{6000}$, the diameter of main xylem vessels in wheat root decreased significantly, exodermal cell began to shrink and endodermal cell shrink slightly (Fig. 5d; 6d), even the endodermis in root of wheat AK58 appeared slightly thickened (Fig. 6d). In addition, cell in exodermis and endodermis of root also shrank when wheat seedlings of XM13 and AK58 were treated with 20\% PEG $_{6000}$ (Fig. 5e; $6 \mathrm{e})$, and the endodermis in root of wheat AK58 showed distinct thick (Fig. 6e), but the necrosis of exodermises was found in seedling root of wheat XM13 (Fig. 5e).

As shown in Fig. 7a and Fig. 8a, in seedling leaf of wheat XM13 and AK58 under the normal condition, the upper epidermis and lower epidermis kept complete, palisade tissue was thicker and the arrangement of its cells were close, and there were a large number of spongy tissue cell arranged in an orderly manner. Under the stress of 5$15 \% \mathrm{PEG}_{6000}$, compared with the control, palisade tissue cells in leaf of wheat XM13 became shorter, the arrangement of spongy tissue was disorder (Fig. 7b, 7c and $7 \mathrm{~d}$ ), while there were no significant changes in leaf of wheat AK58. When the concentration of $\mathrm{PEG}_{6000}$ increased to $20 \%$, palisade tissue cell in seedling leaf arranged loosely, the thickness of spongy tissue decreased, and the arrangement of spongy tissue cell was irregular (Fig. 7e; 8e). Under the stress of $25 \% \mathrm{PEG}_{6000}$, palisade tissue in seedling leaf of two wheat cultivars reduced significantly, spongy tissue cell appeared no regular arrangement (Fig. 7f; $8 \mathrm{f}$ ), indicating that tissue structure in leaf of wheat seedling were destroyed.

\section{Discussion}

It is well known, drought is one of serious environment problems that could influence growth and development of plant. When water is shortage, plant could appear the relative adaptability from morphological structure, physiological and biochemical in order to ensure the relatively stable growth of plant, for example, leaf area reduces and root activity increases (Ashraf, 2010; Mwadzingeni et al., 2016). In this study, the germination rate and germination potential of two wheat cultivars significantly decreased along with the increase of $\mathrm{PEG}_{6000}$ concentration, similar result was also found in citrus (Zaher-Ara et al., 2016). Compared with wheat 


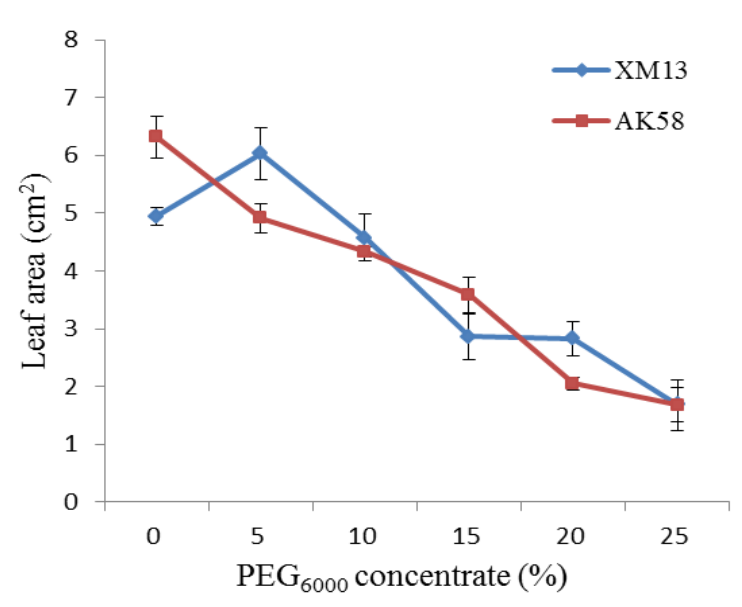

Fig. 4: The leaf area of wheat seedling under drought stress. The error bars represented standard errors

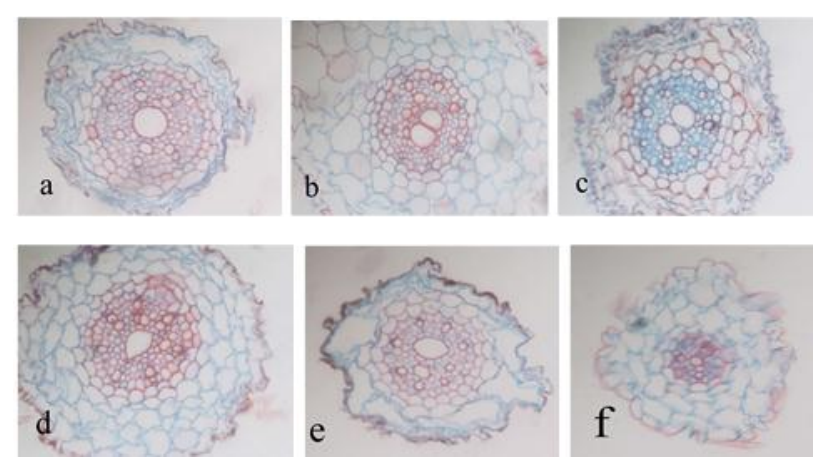

Fig. 5: Paraffin transection of root from wheat XM13 under different drought stress $(\times 200)$. a, b, c, d, e and $f$ represented root from wheat seedlings treated with $0 \%$ $\mathrm{PEG}_{6000}, 5 \% \mathrm{PEG}_{6000}, 10 \% \mathrm{PEG}_{6000}, 15 \% \mathrm{PEG}_{6000}, 20 \%$ $\mathrm{PEG}_{6000}$ or $25 \% \mathrm{PEG}_{6000}$, respectively

AK58, the germination energy of wheat XM13 was greater under the normal condition, yet was significant lower than wheat AK58 under drought stress, and the germination rate of wheat AK58 was higher than wheat XM13 at 25\% $\mathrm{PEG}_{6000}$. Germination energy and germination rate are important indicators to detect the quality of seeds, and strong germination energy and high germination rate indicate the emergence of neat and fast (Farooq et al., 2013; Allahverdiyev, 2015). Thus, the germination of wheat seeds could be inhibited by drought stress, and the drought-resistant ability of wheat AK58 might be stronger than XM13.

Root is the main organ of plant to absorb water and nutrient, could firstly feel effects of water deficits (Comas et al., 2013; Janiak et al., 2015), and plays a key role in response to drought stress. Effects of drought stress on plant root are reflected in many aspects, such as root length and root number. In this study, the formation of wheat root and the length of wheat XM13 root were little
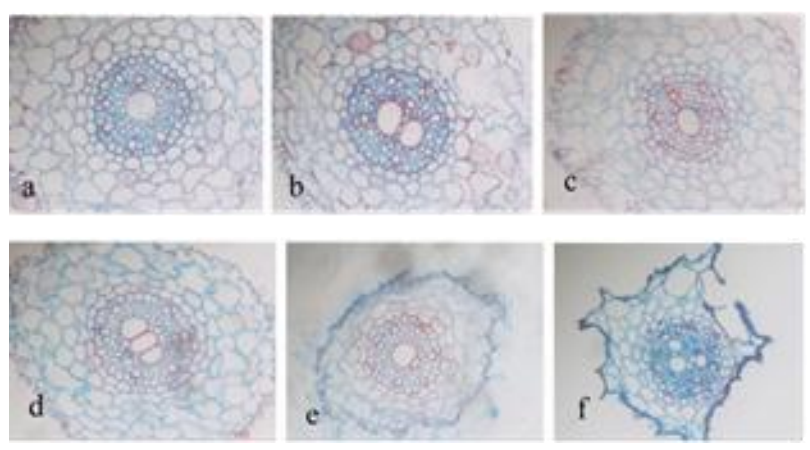

Fig. 6: Paraffin transection of root from wheat AK58 under different drought stress $(\times 200)$. a, b, c, d, e and $f$ represented root from wheat seedlings treated with $0 \%$ $\mathrm{PEG}_{6000}, 5 \% \mathrm{PEG}_{6000}, 10 \% \mathrm{PEG}_{6000}, 15 \% \mathrm{PEG}_{6000}, 20 \%$ $\mathrm{PEG}_{6000}$ or $25 \% \mathrm{PEG}_{6000}$, respectively
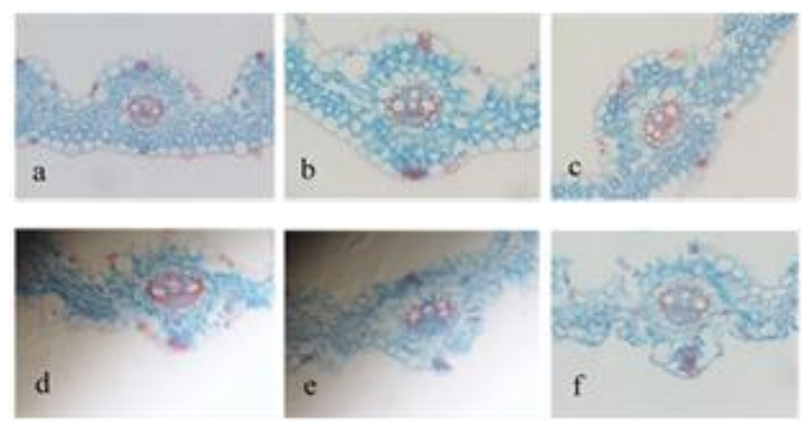

Fig. 7: Paraffin transection of leaf from wheat XM13 under different drought stress $(\times 200)$. a, b, c, d, e and $f$ represented leaf from wheat seedlings treated with $0 \%$ $\mathrm{PEG}_{6000}, 5 \% \mathrm{PEG}_{6000}, 10 \% \mathrm{PEG}_{6000}, 15 \% \mathrm{PEG}_{6000}, 20 \%$ $\mathrm{PEG}_{6000}$ or $25 \% \mathrm{PEG}_{6000}$, respectively
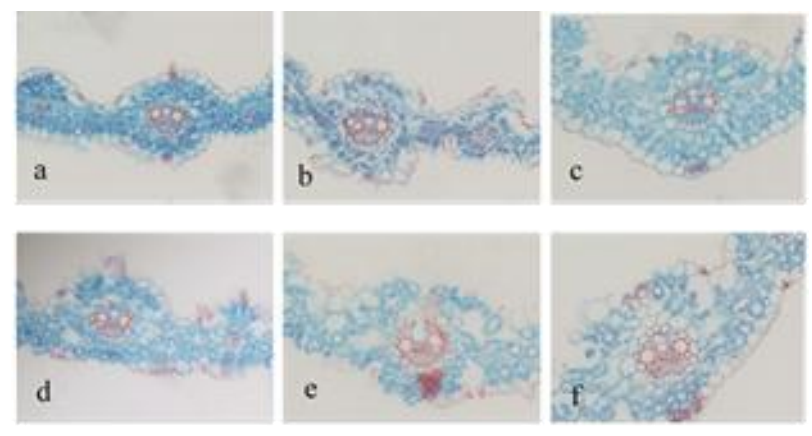

Fig. 8: Paraffin transection of leaf from wheat AK58 under different drought stress $(\times 200)$. a, b, c, d, e and $\mathrm{f}$ represented leaf from wheat seedlings treated with $0 \%$ $\mathrm{PEG}_{6000}, 5 \% \mathrm{PEG}_{6000}, 10 \% \mathrm{PEG}_{6000}, 15 \% \mathrm{PEG}_{6000}, 20 \%$ $\mathrm{PEG}_{6000}$ or $25 \% \mathrm{PEG}_{6000}$, respectively

influenced under drought stress, however root length of wheat AK58 can be promoted, its root was the longest as stressed with $10 \% \quad \mathrm{PEG}_{6000}$ and appeared extremely significant compared with the control. It was also found that 
root growth of baicalensis could be promoted by low concentration of $\mathrm{PEG}_{6000}$ and inhibited at high concentration (Hua and $\mathrm{Li}, 2011$ ). Further study showed that the cell volume of endodermis and exodermis in wheat root had a trend of decline along with the increase of $\mathrm{PEG}_{6000}$ concentration, and then shrink at the stress of $15-25 \%$ $\mathrm{PEG}_{6000}$, but effect of drought stress on root of wheat XM13 was especially significant compared with wheat AK58 (Tang et al., 2014). Moreover, the diameter of main xylem vessels in wheat seedling root also decreased under the stress of $15 \% \mathrm{PEG}_{6000}$, which was especially significant under the high concentration of $\mathrm{PEG}_{6000}$, and the endoderm in root of wheat AK58 showed distinct thick. These results indicated that growth of wheat seedling root was influenced by drought stress, and this effect would be obviously stronger along with the increase of drought degree, especially was significant on root of wheat XM13.

Leaf is the main part of plant photosynthesis which can directly affect the strength of photosynthesis. Under drought stress, leaf would protect water and improve the utilization rate of water in plant by reducing growth rate of leaf, which can effectively reduce the transpiration and loss of water. In this study, leaf area of wheat seedling decreased under drought stress, even was smaller under the stress of $15-25 \% \mathrm{PEG}_{6000}$, which reached significant difference with the control. Under the normal condition, leaf area of wheat AK58 was significantly greater than wheat XM13, but the decreasing amplitude of wheat AK58 leaf area was greater than wheat XM13 under drought stress. It is found in some research that once plant is short of water, cell volume of leaf could be inhibited first, and then leaf area would be smaller (Chen et al., 2012). Further, study identified palisade tissue cell in wheat leaf gradually became shorter and disorder, and the thickness of spongy tissue decreased under drought stress, (Zhang et al., 2015; Han et al., 2016). However, under the stress of $20-25 \%$ $\mathrm{PEG}_{6000}$, the arrangement of palisade tissue cell or spongy tissue cell in wheat leaf was significantly loose and irregular, inferring that this degree of drought stress might be serious to wheat seedling. Furthermore, the atrophy of endodermis cell in leaf of wheat AK58 should be later compared with wheat XM13, which is consistent with structure changes of wheat root under drought stress, thus wheat AK58 seedling might have strong adaptability to drought stress compared with wheat XM13.

\section{Conclusion}

In this study, it was found that germination, root and leaf growth of wheat cultivars were considerably influenced by drought stress, increasing drought stress level would obviously affect stronger cv. XM13 than cv. AK58. However, the defense response of plant under drought stress is very complicated, further research is needed to explore the mechanism of wheat seedling in response to drought stress.

\section{Acknowledgments}

This work was supported by the National Science-technology Support Plan Projects (2013BAD07B14) and Project for Innovative Talent of Science and Technology in University of Henan Province (17HASTIT034) in P. R. China.

\section{References}

Allahverdiyev, T., 2015. Effect of drought stress on some physiological traits of durum (Triticum durum Desf.) and bread (Triticum aestivum L.) wheat genotypes. J. Stress Physiol. Biochem., 11: 30-32

Ashraf, M., 2010. Inducing drought tolerance in plants: recent advances. Biotechnol. Adv., 28: 169-183

Bhargava, S. and K. Sawant, 2013. Drought stress adaptation: metabolic adjustment and regulation of gene expression. Plant Breed., 132: 2132

Carvalho, P., S Azam-Ali and M.J. Foulkes, 2014. Quantifying relationships between rooting traits and water uptake under drought in Mediterranean barley and durum wheat. J. Integr. Plant Biol., 56: 455-469

Chen, X., Y.F. Xu and Z.Y. Zhang, 2012. Leaf anatomical structure and photosynthetic physiology responses of Sorbus folgneri seedlings under drought stress. Acta Bot. Boreali-Occidentalia Sin., 32: 111116

Comas, L.H., S.R. Becker, V.M.V. Cruz, P.F. Byrne and D.A. Dierig, 2013. Root traits contributing to plant productivity under drought. Front. Plant Sci., 4: 442

Cseuz, L., J. Pauk, C. Lantos and I. Kovacs, 2009. Wheat breeding for drought tolerance. (Efforts and results.). Alps-Adria Sci. Workshop, 36: $245-248$

Boscaiu, M., I. Bautista, P. Donat, A. Lidon, J. Llinares and C. Lull, 2011. Plant responses to abiotic stress. Curr. Opin. Biotechnol., 22: S130 S130

Duan, H.Y., X.S. Ding, J.Y. Song, Z.K. Duan, Y.Q. Zhou and C.E. Zhou, 2009. Effects of kanamycin on growth and development of Arabidopsis thaliana seedling, cotyledon and leaf. Pak. J. Bot., 41: $1611-1618$

El Harfi, M., H. Hanine, H. Latrache, A. Nabloussi, 2016. Effect of drought and salt stresses on germination and early seedling growth of different color-seeds of sesame (Sesamum indicum). Int. J. Agric. Biol., 18: 1088-1094

Farooq, M., M. Irfan, T. Aziz, I. Ahmad and S.A. Cheema, 2013. Seed priming with ascorbic acid improves drought resistance of wheat. $J$. Agron. Crop Sci., 199: 12-22

Gong, D., 2014. Studies on physiological indexes of drought resistance in wheat. Master Degree Thesis, pp: 1-8. Yantai University, Shandong Sheng, China

Grzesiak, S., M.T. Grzesiak, T. Hura, L. Marcinska and A. Rzepka, 2013. Changes in root system structure, leaf water potential and gas exchange of maize and triticale seedlings affected by soil compaction. Environ. Exp. Bot., 88: 2-10

Han, Y.P., Y.L. Li, Z.H. Lei, D. Zhao and X.S. Jia, 2016. Influence of different root temperature treatment on tomato leaves microstructure. Northern Hortic., 3: 17-19

Hossain, A., M.A.Z. Sarker, M. Saifuzzaman, J.A. Silva, M.V. Lozovskaya and M.M. Akhter, 2013. Evaluation of growth, yield, relative performance and heat susceptibility of eight wheat (Triticum aestivum L.) genotypes grown under heat stress. Int. J. Plant Prod., 7: 615-636

Hua, Z.R. and X.L. Li, 2011. Effect of peg treatment on seed germination and seedling growth of Shangluo scutellaria baicalensis. Seed, 41: $1338-1353$

Hu, H. and L. Xiong, 2014. Genetic engineering and breeding of droughtresistant crops. Plant Biol., 65: 715-741

Janiak, A., M. Kwasniewski and I. Szarejko, 2015. Gene expression regulation in roots under drought. J. Exp. Bot., 67: 142-147 
Jung, H., K. Lee and W. Chun, 2006. Statistical models and evaluation of tolerances in national rules for the germination test of crop seed testing. J. Biomath., 21: 1365-1368

Khodarahmpour, Z. and M. Motamedi, 2001. Evaluation of drought and salinity stress effects on germination and early growth of two cultivars of maize (Zea mays L.). Afr. J. Biotechnol., 10: 14868-14872

Lipiec, J., C. Doussan, A. Nosalewicz and K. Kondracka, 2013. Effect of drought and heat stresses on plant growth and yield: a review. Int. J. Agrophys., 27: 463-477

Liu, X.F., X.F. Zhu, Y.Z. Pan, S.S. Li, Y.X. Liu and Y.Q. Ma, 2016. Agricultural drought monitoring: progress, challenges, and prospects. J. Geogr. Sci., 26: 750-767

Lopes, M.S. and M.P. Reynolds, 2010. Partitioning of assimilates to deeper roots is associated with cooler canopies and increased yield under drought in wheat. Funct. Plant Biol., 37: 147-156

Ma, L.J., L.L. Wang, Y.X. Mei, S.W. Zhang, W. Wei, J.Y. Wang and Y.L. Zhang, 2016. Cross adaptation tolerance in rice seedlings exposed to PEG induced salinity and drought stress. Int. J. Agric. Biol., 18: 535541

Maqsood, M., M.A. Shehzad, S. Ahmad and S. Mushtaq, 2012. Performance of wheat (Triticum aestivum L.) genotypes associated with agronomical traits under water stress conditions. Asian J. Pharm. Biol. Res., 2: 45-50

Maqsood, M., M.A. Shehzad, A. Wahid and A.A. Butt, 2013. Improving drought tolerance in maize (Zea mays) with potassium application in furrow irrigation systems. Int. J. Agric. Biol., 15: 1193-1198

Mwadzingeni, L., H. Shimelis, E. Dube, M.D. Laing and T.J. Tsilo, 2016. Breeding wheat for drought tolerance: progress and technologies. $J$. Integr. Agric., 15: 935-943

Peleg, Z., T. Fahima, A.B. Korol, S. Abbo and Y. Saranga, 2011. Genetic analysis of wheat domestication and evolution under domestication. J. Exp. Bot., 62: 5051-5061

Ramya, R., G.P. Singh, N. Jain, P.K. Singh, M.K. Pandey and K. Sharma, 2016. Effect of recurrent selection on drought tolerance and related morpho-physiological traits in bread wheat. PloS One, 11: e0156869
Shen, Y.F., S.Q. Li and M.A. Shao, 2013. Effects of spatial coupling of water and fertilizer applications on root growth characteristics and water use of winter wheat. J. Plant Nutr., 36: 515-528

Tang, Y.J., M. Meng, X.P. Deng, C.Q. Tang, R. Deng and S.S. Yang, 2014. Relationship between drought resistance and root characteristics of wheat under drought stress. J. Northwest $A \&$ \& $F$ Univ., 42: 48-60

Wang, C.Y., W.X. Liu, Q.X. Li, D.Y. Ma, H.F. Lu, W. Feng, Y.X. Xie, Y.J. Zhu and T.C. Guo, 2014a. Effects of different irrigation and nitrogen regimes on root growth and its correlation with above-ground plant parts in high-yielding wheat under field conditions. Field Crops Res., 165: 138-149

Wang, Y., Z.S. Liang, C.M. Gong, R.L. Han and J. Yu, 2014b. Effect of drought on leaf anatomical characteristics of four Artemisia species in the Loess Plateau. Acta Ecol. Sin., 34: 4535-4548

Yan, M., 2015. Seed priming stimulate germination and early seedling growth of Chinese cabbage under drought stress. S. Afr. J. Bot., 99: 88-92

Yu, X.R., B. Li, L.L. Wang, X.Y. Chen, W.J. Wang, Y.J. Gu, Z. Wang and F. Xiong, 2016. Effect of drought stress on the development of endosperm starch granules and the composition and physicochemical properties of starches from soft and hard wheat. J. Sci. Food Agric., 96: $2746-2754$

Zaher-Ara, T., N. Boroom and M. Sadat-Hosseini, 2016. Physiological and morphological response to drought stress in seedlings of ten citrus. Trees, 30: 985-993

Zalibekov, Z.G., 2011. The arid regions of the world and their dynamics in conditions of modern climatic warming. Arid Ecosys., 1: 1-7

Zhang, J.J., B. Dell, W.J. Ma, V. Rudy, X.M. Zhang and T. Oteri, A. Foreman and D. Laird, 2016. Contributions of root WSC during grain filling in wheat under drought. Front. Plant Sci., 7: 1-9

Zhang, L.L., Y. Shi, X. Qi, Q.X. Wang and L. Cui, 2015. Effects of drought stress on the ultrastructure and physiological indexes of leaf cells in three potato varieties. Agric. Res. Arid Areas, 33: 75-80

(Received 08 March 2017; Accepted 22 May 2017) 